\title{
Outcomes of a chronic heart failure training program for health professionals
}

\author{
Rosemary O Higgins ${ }^{1}$, Hema S Navaratnam ${ }^{1}$, Barbara M Murphy ${ }^{1,}$, Sandra Walker ${ }^{3}$, Marian UC \\ Worcester $^{1,2}$
}

1. Heart Research Centre, Melbourne, Australia. 2. Department of Psychiatry, The University of Melbourne, Australia. 3 School of Population Health, The University of Melbourne, Australia.

Correspondence: Rosemary Higgins. Address: Senior Research Fellow, Heart Research Centre, PO Box 2137. The Royal Melbourne Hospital 3050, Victoria, Australia. Email: rosemary.higgins@heartresearchcentre.org.

Received: September 3, 2012

DOI : $10.5430 /$ jnep.v3n7p68
Accepted: October 18, 2012

URL: http://dx.doi.org/10.5430/jnep.v3n7p68

Online Published: January 11, 2013

\section{Abstract}

The recent proliferation of chronic heart failure (CHF) disease management programs has created a need for greater provision of high quality training for health professionals in this area. Greater practitioner self-efficacy and positive practice change are both indicators of training impact. This study investigated the effects of health professional training by examining health professionals' self-efficacy and practice changes. In addition, perceived barriers to setting up a program for CHF patients were explored. A sample of 46 health professionals completed a questionnaire prior to and immediately after participation in the training program. Telephone interviews with participants were undertaken four months after training. Participants' self-efficacy significantly improved over the course of the training. This improvement was maintained four months later. Positive practice changes included translating knowledge into clinical practice, changing or setting up a program, improving patient management and education, implementing clinical pathways and using action plans. The major barriers experienced when setting up a program were limited funding and insufficient staff. One of the main highlights of this study was the increased self-efficacy of practitioners over the four month period, indicating the positive boost professional training can provide. This finding highlights participants' motivation to change professional practice and to set goals for practice change. More CHF training programs need to be developed and a stronger emphasis towards evaluating these programs is necessary to ensure a prepared and proactive workforce in this area.

\section{Key words}

Chronic heart failure, Health professional training programs, Practice change, Self-efficacy, Perceived barriers

\section{Introduction}

Chronic heart failure (CHF) currently affects 277,800 Australians ${ }^{[1]}$, with about 30,000 new cases diagnosed annually ${ }^{[2,3]}$. Integrated disease management care provided by specialist nurses and multidisciplinary teams has been shown to improve outcomes for patients with CHF, significantly reducing the number of unplanned readmissions, length of hospital stay, hospital costs, and mortality ${ }^{[2,4]}$. Patients with CHF require a rehabilitation program that meets the specific needs of their condition. In Victoria, Australia, such programs have been developed and implemented in a range of hospital and community settings. However, the application of evidence-based knowledge is often less than optimal in the practice and 
care of patients with $\mathrm{CHF}^{[4]}$. As such, there is a recognised need for greater integrated disease management for CHF patients through the provision of education, comprehensive discharge planning and home visits.

The Integrated Disease Management for Chronic Heart Failure training program (CHF training program) was developed by the Heart Research Centre (HRC) in collaboration with an expert steering committee. The program was developed in response to requests from service providers for evidence-based training for practitioners working with CHF patients ${ }^{[4]}$. The program was designed to build the capacity of health professionals to understand and respond to the needs of clients with CHF. The CHF training program is a three-day course, which has been delivered annually by the HRC since 2004 . The program attracts a range of health professionals. To date, 258 health professionals have undertaken the training. The program objectives can be divided into three categories: first, to expand participants' knowledge of CHF; second, to improve the management of CHF; and third, to provide skills related to program development. Once training programs such as this are established, it is important that a rigorous evaluation of the program is conducted.

While participant satisfaction provides some information about the suitability of training, goal based outcome evaluations provide a good measure of program effectiveness ${ }^{[5]}$. Training program outcomes are often measured by the knowledge gained by participants during the program ${ }^{[6-10]}$. However, gains in knowledge as a result of attending a training course are difficult to measure, particularly when many of the participants are professionals with an existing knowledge base. Whilst knowledge is an important aspect of training, it has been suggested that other outcomes such as confidence to apply knowledge in the workplace are equally, if not more, important ${ }^{[11-13]}$.

The concept of self-efficacy offers an in-depth measure of the influence of training on work practice. Self-efficacy refers to beliefs regarding one's ability to perform a defined task in a specific situation ${ }^{[14]}$. The task specificity of self-efficacy distinguishes it from self-confidence, which is a stable, generalised personality characteristic. Self-efficacy has been observed as a predictor of professional practice behaviours and as an outcome of clinical training ${ }^{[14]}$. Self-efficacy has been studied in the training of health professionals ${ }^{[11,15,16]}$. In each study, increases in health professional self-efficacy were measured following training completion. Training also needs to support the translation of increased self-efficacy into positive practice change. Positive practice change has been found to contribute to improved patient outcomes as a result of professional training ${ }^{[11]}$. Knowledge of participants' intended and actual practice change provides information about both the influence of training on work practice and the barriers to practice change.

\section{Aims}

The aims of the present evaluation were: 1) to determine changes in participants' self-efficacy after attending the program; 2) to identify participants' intended and actual changes in professional practice after the training program; 3) to identify barriers to practice change experienced by training participants and 4) to investigate the relationships between selfefficacy, barriers and practice change.

\section{Methods}

\subsection{Participants}

Forty-six health professionals attended one of two 3-day training programs held in Melbourne, Australia. Prior to the training, all were invited and agreed to participate in the study. Forty-five completed the pre-program questionnaire, 42 completed the post program questionnaire and 44 participated in a 4 month follow up interview. All completed at least two assessments.

\subsection{Procedure}

Participants completed a pre-program questionnaire immediately prior to starting training. The pre-program questionnaire comprised items regarding participants' self-efficacy and expectations about program attendance. Demographic and 
professional details were also collected at this time. Completed questionnaires were collected by a researcher prior to the commencement of training.

Participants completed a post program questionnaire immediately following the training. The post-program questionnaire comprised items regarding self-efficacy and intended practice change. Completed post-program questionnaires were placed in a designated box at the program venue.

Participants were contacted by telephone four months after the training program to participate in a follow-up interview. The interview addressed changes made in work practice since attending the program. It also covered barriers encountered in implementing practice change and ways of overcoming such barriers.

\subsection{Measures}

\subsubsection{Self-efficacy}

Self-efficacy was measured using a 16-item scale concerning aspects related to work practice. The measure was adapted from a self-efficacy scale devised by Lorenz and colleagues ${ }^{[11]}$. Items included questions about confidence regarding disease specific knowledge, management of CHF; and confidence regarding program development skills. Responses were recorded on a 5-point Likert scale ranging from $1=$ not at all confident to $5=$ very confident in performing the task. The possible score range was from 16 to 80, with low scores indicating lower self-efficacy. Similar questions have been used in past evaluations of a chronic disease self-management training program ${ }^{[15]}$. Internal consistency of the measure is high with a Cronbach’s alpha of .922. A copy of the questionnaire is available from the authors upon request.

\subsubsection{Intended and actual practice change}

Intended and actual practice changes were assessed immediately after the training program and four months later. Participants were asked open ended questions regarding the changes they intended to make in their usual practice with CHF patients and changes made at the workplace.

\subsubsection{Barriers to practice change}

The barriers encountered in developing and implementing CHF programs were ascertained using a list of possible barriers (see Table 3) which participants rated from 1 = not at all relevant to 5 = highly relevant. Corresponding open-ended questions were used to assess participants’ plans to overcome barriers.

\subsubsection{Socio-demographic characteristics}

Details were also collected regarding participants' gender, age, profession, qualifications, employment status, current involvement in a CHF program and source of funds for training.

\subsection{Data analysis}

Both qualitative and quantitative analyses were undertaken. For most responses, frequencies were calculated. Changes over time in self-efficacy were evaluated using repeated measures analysis of variance (ANOVA). Correlations between self-efficacy, perceived barriers and practice changes were calculated. Qualitative data from open-ended questions were analysed thematically, with responses coded into categories to represent specific themes.

\section{Results}

\subsection{Participant characteristics}

Most participants were female and nurses. A minority were physiotherapists, dieticians and speech pathologists. They ranged in age from 24 to 65 years with most aged between 30-39 years (44\%). Most were highly qualified. Just under half 
(45.7\%) were currently employed in a CHF program. In terms of funding to attend the training, just over half of the participants had been funded by their employer. The remainder were either fully or partially self-funded. Demographic and professional characteristics of participants are shown in Table 1.

Table 1. Demographic and professional characteristics of participants in the CHF training program

\begin{tabular}{ll}
\hline Characteristic & $\mathbf{n}(\mathbf{\%}) \mathbf{( N = 4 0 )}$ \\
\hline Gender & $1(2.2)$ \\
Male & $45(97.8)$ \\
Female & \\
Age & $26(56.5)$ \\
$20-39$ & $20(43.5)$ \\
$>40$ & \\
Profession & $38(82.6)$ \\
Nursing & $6(13.0)$ \\
Physiotherapy & $1(2.2)$ \\
Dietetics & $1(2.2)$ \\
Speech pathology & \\
Qualifications & $10(22.7)$ \\
Basic professional/hospital training & $13(29.5)$ \\
Professional certificate & $21(47.7)$ \\
Post graduate & \\
Employment status & $20(43.5)$ \\
Full time & $24(52.2)$ \\
Part time & $2(4.3)$ \\
Casual & \\
\hline
\end{tabular}

\section{Changes in participants' self-efficacy over time}

Participants' self-efficacy mean (SD) scores at pre-program, post-program and the 4 month follow-up were 45.59 (10.79), 60.82 (8.74) and 61.09 (8.75) respectively. Overall, self-efficacy improved significantly over the 4 month period $(\mathrm{F}(2,38)$ $=81.61, p<.001$ ), with an effect size of .811. All of the improvement occurred from the pre to post program assessments $(\mathrm{F}(1,41)=142.44, p<.001$; effect size of .776). There was no further change in self-efficacy from post training to 4 months $(\mathrm{F}(1,39)=0.19, p=.663$; effect size of .005$)$. Mean self-efficacy scores for the three time-points are shown in Figure 1.

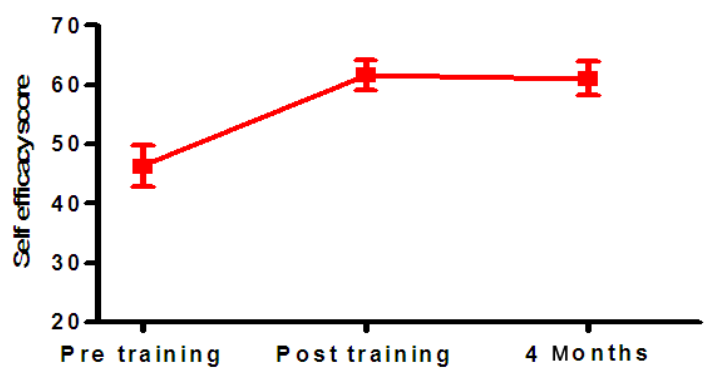

Figure 1. Mean self-efficacy scores at pre-program, post-program and the 4 month follow-up

\section{I ntended and actual practice change}

After the training, most participants intended to change their work practice (see Table 2). Around a quarter ( $\mathrm{n}=11 ; 26.2 \%)$ intended to improve patient management and education with slightly less than a quarter intending to establish a new program or change an existing program $(n=10 ; 23.8 \%)$. These changes were generally carried out as intended. About a 
fifth intended to adopt action plans $(n=9 ; 21.4 \%)$ and apply behavioural strategies ( $n=8 ; 19.0 \%)$, although few actually carried out the latter. No participants indicated an intention to apply the knowledge learned in training or to network with other health professionals. Interestingly, however, these were the most common changes made when participants returned to their workplace. Changes not carried out as intended included reviewing patients' medications, modifying interviewing techniques, identifying patient barriers to change, improving patient care and implementing clinical outcomes. Other practice changes were reported by only a small minority as shown in table 2 .

Table 2. Intended and actual practice change

\begin{tabular}{lll}
\hline Theme & $\begin{array}{l}\text { Intended } \\
\mathbf{n}(\mathbf{\%})\end{array}$ & $\begin{array}{l}\text { Actual } \\
\mathbf{n}(\%)\end{array}$ \\
\hline Improve patient management \& education & $11(26.2)$ & $8(22.2)$ \\
Set up/change program & $10(23.8)$ & $9(25.0)$ \\
Adopt action plans & $9(21.4)$ & $6(16.7)$ \\
Apply behavioural strategies & $8(19.0)$ & $1(2.8)$ \\
Implement clinical pathways & $7(16.7)$ & $6(16.7)$ \\
Increase GP liaison & $5(11.9)$ & $4(11.1)$ \\
Review patients' medications & $3(7.14)$ & $0(0)$ \\
Educate staff & $2(4.76)$ & $2(5.6)$ \\
Apply knowledge & $0(0)$ & $16(44.4)$ \\
Networking and support & $0(0)$ & $3(8.3)$ \\
Other & $7(16.7)$ & $0(0)$ \\
\hline
\end{tabular}

Note. $\mathrm{N}=42$ for changes intended at post program, $\mathrm{N}=36$ for actual change at 4 months. Missing data due to incomplete questionnaire; GP = General Practitioner or Family Doctor .

\section{Barriers to setting up a CHF rehabilitation program}

The main barriers experienced when setting up a CHF program were financial difficulties and insufficient trained staff. A handful of participants reported a lack of support and insufficient patients as barriers. Reported barriers are shown in table 3.

Table 3. Barriers experienced in setting up CHF rehabilitation programs

\begin{tabular}{llcl}
\hline Barrier & $\begin{array}{l}\text { Highly relevant } \\
\mathbf{n}(\%)\end{array}$ & $\begin{array}{l}\text { Some relevance } \\
\mathbf{n}(\%)\end{array}$ & $\begin{array}{l}\text { Not relevant } \\
\mathbf{n}(\%)\end{array}$ \\
\hline Inadequate finance & $14(66.7)$ & $4(19.1)$ & $3(14.3)$ \\
Insufficient trained staff & $12(57.1)$ & $8(38.1)$ & $1(4.8)$ \\
Lack of doctor support & $5(23.8)$ & $11(52.3)$ & $5(23.8)$ \\
Lack of other support & $3(14.3)$ & $12(57.1)$ & $6(28.5)$ \\
Insufficient patients & $2(10.0)$ & $9(45.0)$ & $9(45.0)$ \\
\hline
\end{tabular}

Note. $\mathrm{N}=21$ except barrier of insufficient patient where $\mathrm{N}=20$ due to incomplete questionnaire. Only participants from one training program were asked about barriers. Percentages for all sources $>100$ because participants ticked $>1$ option.

\section{Relationship between self-efficacy, perceived barriers and practice change}

There was a significant negative correlation between the number of reported barriers and the number of practice changes reported at the 4 month follow-up $(r=-.698, p=.001)$. Fewer barriers were associated with more practice changes. There was also a significant negative correlation between the number of reported barriers and self-efficacy scores at post program $(r=-.447, p=.048)$, indicating that higher self-efficacy was associated with fewer perceived barriers. However, the number of reported barriers was not significantly correlated with self-efficacy scores at either pre-program $(r=-.269$, $p=.251)$ or the 4 month follow up ( $r=-.270, p=.263)$. The number of practice changes made by 4 months was not significantly related to self-efficacy at either pre-program, post program or 4 months $(r=-.044, p=.80 ; r=.08, p=.65 ; r$ $=.038, p=.827$ ) respectively. 


\section{Discussion}

This study demonstrated that participants' self-efficacy improved immediately after the training program with improvements maintained at four months. This finding is consistent with previous research suggesting that professional training increases confidence in participants' work practice ${ }^{[6,11-13,15]}$. That self-efficacy increased and was maintained after the training program demonstrates that the program was designed and administered in such a way that participants were able to increase their confidence in undertaking a range of tasks related to CHF rehabilitation at completion of the program.

Intentions regarding practice change were somewhat different from participants' reported practice changes at the four month follow up. Interestingly, the least frequently listed intention of applying knowledge learnt from the training program was the change most commonly reported at 4 months. Other intended changes, such as integrating behavioural strategies and reviewing medication, were not evident when actual changes were assessed. One reason for this finding could be that the training insufficiently skilled participants to implement these changes although it appeared to make them aware of the need for such strategies to be adopted.

\section{I mplications}

The CHF training program utilised an interprofessional learning approach creating opportunities for participants to exchange ideas. It would be interesting to know whether practice changes translated to increased self-efficacy and better patient outcomes. A future study should be undertaken to address this question.

\section{Limitations and future research}

The study had a number of limitations, including a relatively small sample size which limited comparisons across groups. Increased sample size would provide a better understanding of how a range of participants experienced the training program. More specifically, future research could investigate how different health professional groups respond to such training.

To determine whether practice changes occurred as result of the training would require a control group in a randomised control trial. More stringent data collection would also enhance the validity of results. For example, a mix of self-report and observational data collection would further enrich the data. Employing direct observation of participants at their workplace would provide the opportunity to record practice changes without having to depend only on participants' self-report data.

Despite these limitations, information was successfully collected from most of the participants at the three different time points. This highlights that effective collaboration between researchers, trainers and participants has proven to be beneficial.

\section{References}

[1] AIHW 2011. Cardiovascular Disease: Australian facts 2011.Cardiovascular disease series no. 35.Cat. no. CVD 53. Canberra:AIHW.

[2] Australian Institute of Health and Welfare. Cardiovascular Disease: Australian Facts 2011. Canberra: Australian Institute of Health and Welfare; 2011.

[3] Field B. Heart failure What of the future? Canberra: Australian Institute of Health and Welfare; 2003.

[4] HARP CHF Working Party. Hospital admission risk program (HARP): Chronic heart failure working party report. Melbourne: Victorian Government Department of Human Services; 2003.

[5] Posavac EJ, Carey RG. Program evaluation methods and case studes (5th ed). New Jersey: Prentice Hall; 1997. PMid:9107000 
[6] Filippini A, Sessa A, Di Giuseppe G, Angelillo IF. Evidence-based practice among nurses in Italy. Evaluation and the Health Professions. 2011; 34(3): 371-82. PMid:21138912 http://dx.doi.org/10.1177/0163278710387924

[7] Ford M, Martin RD, Hilton LW, Ewert-Flannagan T, Corrigan GK, Johnson G, et al. Outcomes study of a course in breast-cancer screening. Journal of Cancer Education. 1997; 12(3): 179-84. PMid:9376257

[8] Kaye W, Mancini ME, Rallis SF. Advanced cardiac life support refresher course using standardized objective-based Mega Code testing. Critical Care Medicine. 1987; 15(1): 55-60. PMid:3792016 http://dx.doi.org/10.1097/00003246-198701000-00013

[9] Payne JM, France KE, Henley N, D'Antoine HA, Bartu AE, O'Leary CM, et al. RE-AIM evaluation of the Alcohol and Pregnancy Project: educational resources to inform health professionals about prenatal alcohol exposure and fetal alcohol spectrum disorder. Evaluation and the Health Professions. 2011; 34(1): 57-80. PMid:21292723 http://dx.doi.org/10.1177/0163278710381261

[10] Qureshi KA, Gershon RR, Merrill JA, Calero-Breckheimer A, Murrman M, Gebbie KM, et al. Effectiveness of an emergency preparedness training program for public health nurses in New York City. Family \& Community Health. 2004; 27(3): 242-9. http://dx.doi.org/10.1097/00003727-200407000-00011

[11] Lorenz R, Gregory RP, Davis DL. Utility of a brief self-efficacy scale in clinical training program evaluation. Evaluation \& the Health Professions. 2000; 23(2): 182-93. http://dx.doi.org/10.1177/016327870002300204

[12] Manojlovich M. Promoting nurses' self-efficacy: a leadership strategy to improve practice. Journal of Nursing Administration. 2005; 35(5): 271-8. PMid:15891491

[13] Pololi LH, Potter S. Behavioral change in preventive medicine. An efficacy assessment of a physician education module. Journal of General Internal Medicine. 1996; 11(9): 545-7. PMid:8905505 http://dx.doi.org/10.1007/BF02599603

[14] Bandura A. Self-efficacy: toward a unifying theory of behavioral change. Psychological Review. 1977; 84(2): 191-215. PMid:847061 http://dx.doi.org/10.1037/0033-295X.84.2.191

[15] Higgins R, Murphy B, Worcester M, Daffey A. Supporting chronic disease self management: Translating policies and principles into clinical practice. Australian Journal of Primary Health. 2012; 18: 80-7. PMid:22394667 http://dx.doi.org/10.1071/PY11006

[16] Smarr KL. The effects of arthritis professional continuing education in vocational rehabilitation: PhD thesis. University of Missouri - Columbia; 2003. 